\title{
Long, narrow all-light atom guide
}

\author{
Y. Song, D. Milam, and W. T. Hill III \\ Institute for Physical Science and Technology and Laboratory for Atomic, Molecular and Optical Science and Engineering, \\ University of Maryland, College Park, Maryland 20742
}

Received June 9, 1999

\begin{abstract}
A 1-mm-diameter all-light atom guide capable of transporting ultracold atoms tens of centimeters with high efficiency is described. We made the atom tunnel, a dark hollow beam that is blue detuned from resonance, by passing a few tens of milliwatts of power from a TE $M_{00}$ diode laser beam through an optical sequence composed of three axicons and a simple lens. We demonstrate transport of $10^{8} \mathrm{Cs}$ atoms approximately $20 \mathrm{~cm}$ with minimal heating. We show that it is possible for one to control the direction and speed of the atoms in the tunnel by varying the detuning of the tunnel beam. (c) 1999 Optical Society of America

OCIS codes: $020.0020,020.7010$.
\end{abstract}

One of the more promising proposals for guiding and focusing neutral atoms involves dark hollow laser beams. ${ }^{1-4}$ When the frequency of the laser is blue detuned from resonance, the dipole force that the atoms experience in the light confines them to the dark core of the beam, where they can be transported with a minimal interaction with the light. Dark hollow beams, which are all-light guides, show promise for long-distance transport of large numbers of ultracold atoms, making them a strong competitor with hollowcore fiber atom guides. ${ }^{5}$ The advantages that all-light guides have over fiber guides are the ease with which they can be loaded, the elimination of physical walls, and an ability to vary their core size. The last of these makes all-light guides potentially useful for focusing neutral atoms. Realizing these all-light guides should lead to a versatile tool for atom lithography, atom interferometry, and atomic spectroscopy as well as a means for transporting and manipulating B ose- Einstein condensates. Furthermore, since atoms transported in all-light guides do not come into contact with matter, they could be used to transport antimatter as well.

In this Letter we describe a simple way to construct an all-light atom tunnel. In this demonstration we transported clouds of $\sim 10^{8} \mathrm{Cs}$ atoms through an 18-cm-long, 1-mm-diameter core at selected group velocities, which are controllable by the laser detuning. The guide was generated with a series of axicons (each with a $3^{\circ}$ base angle) and a simple lens, as shown in the top inset of Fig. 1. Sixty milliwatts of $\mathrm{TEM}_{00}$ mode diode laser power, blue detuned from the $C$ s resonance line at $852 \mathrm{~nm}$, served as the incident beam. A hollow beam generated with an axicon and a lens has different focal points for the inner and outer walls. Two additional axicons are required for control of the core diameter and wall thickness, as shown in the top inset in Fig. 1. An important byproduct of this arrangement is that most of the diffraction originating from the tip of the first axicon is located outside the core, as shown in the bottom inset of Fig. 1. The main part of Fig. 1 shows that guides generated with axicons have steep walls and dark cores (intensity approximately zero) that extend over most of the diameter. This is an important distinction between this and others means for generating hollow beams from TEM $\mathrm{M}_{00}$ modes. ${ }^{6}$ Typically, holograms, ${ }^{3}$ vor tex gratings, ${ }^{7}$ and hollow-core fibers ${ }^{8}$ produce a low-order doughnut mode that is approximately a TEM ${ }_{01}^{*}$ mode. At best, the intensity of the TEM ${ }_{01}{ }^{*}$ modes vanishes only at the center. At the same time, axicons are more readily available than holograms, vortex gratings, or hollowcore fibers. However, it should be recognized that, in general, Hermite-Gaussian (or Laguerre-Gaussian) modes are not generated with axicons. Consequently, the guide beam generally does not propagate indefinitely, nor is the dark core preserved when the beam is focused with a simple lens. Nevertheless, a tunnel beam that is usable for tens of centimeters can be produced routinely.

To demonstrate the capability of the guide for transporting atoms we used it to guide cold $(\approx 70-140-\mu \mathrm{K})$ Cs atoms. The source of cold atoms was a vaporloaded Cs magneto-optical trap ${ }^{9}$ (MOT) containing

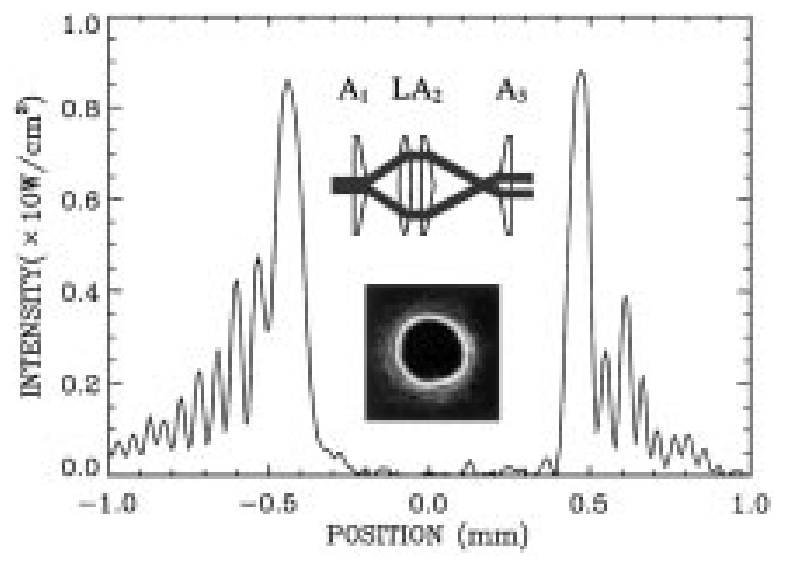

Fig. 1. 1-mm hollow-core beam intensity distribution at the position of the MOT (graph and bottom inset) and optical components for hollow-core beam generation (top inset). Three axicons $\left(A_{1}-A_{3}\right)$, each with a $3^{\circ}$ base angle and $a$ 400-mm focal-length lens ( $L$ ), were employed to generate the hollow core. The spacing between the elements, from left to right, was 44,5 , and $55 \mathrm{~cm}$. The incident $\mathrm{TEM}_{00}$ beam was diffraction limited, with a divergence of $\sim 3 \mathrm{mrad}$ and a spot size of $\sim 2.3 \mathrm{~mm}$ at $A_{1}$. 
$\sim 10^{9}$ atoms in the shape of an ellipsoid, with a major axis two to three times longer than the minor axis. (We achieved this shape by making two of the MOT beams intersect at less than $45^{\circ}$.) The guide beam and the major axis of the MOT were aligned and oriented in the vertical direction. This allowed the cold atoms to fall under gravity while being guided by the tunnel. Forming the MOT inside the tunnel guide facilitated loading (this is possible when the tunnel beam is detuned at least $100 \mathrm{MHz}$ from resonance). Whereas it was necessary to employ saturated absorption spectroscopy to lock the MOT beams to their proper frequencies, the tunnel beam needed only to be stationary to within tens of megahertz, rendering locking unnecessary. We transferred the atoms to the tunnel by turning the MOT beams off. We monitored the evolution of the cloud of atoms (size, location, and density) by the absorption shadow that the cloud of atoms cast on a CCD camera. The beam that was used to form the shadow was split from the MOT trapping beam. The frequency of the beam that formed the shadow was shifted closer to resonance with two acousto-optic modulators that also served as fast shutters, permitting precise timing of the cloud's evolution. We obtained a quantitative measure of the number of atoms in the guide by digitizing the CCD images. Shadow images were taken in both the transverse [Fig. 2(a)] and the longitudinal [Fig. 2(b)] directions with respect to the guide.

The asymmetric shapes of the clouds in the transverse images [Fig. 2(a)] indicate transverse confinement, whereas in the longitudinal direction the atoms exhibit nearly free expansion. One consequence of nearly free expansion is minimal heating. Nevertheless, the atoms do interact with the tunnel at these short times. Figure 3 shows that the position of the center of mass of the cloud in time depends on the detuning. Specifically, the acceleration of the cloud depends inversely on the detuning. It is straightforward to show that the center of mass of the doud in Fig. 2(a) has an acceleration of $\sim 15 \mathrm{~m} / \mathrm{s}^{2}$, appreciably larger than $\mathrm{g}\left(\approx 9.8 \mathrm{~m} / \mathrm{s}^{2}\right)$. We also find that when the guide beam is directed against gravity and detuned by less than $1 \mathrm{GHz}$ it is possible to levitate the center of mass of the cloud, as shown in the top traces in Fig. 3. This ability to control the acceleration gives the experimenter a knob for varying the velocity of the atoms in the guide. The acceleration is a result of recoil during absorption of photons at the guide walls and is proportional to the recoil velocity and the rate at which photons are absorbed. At large detunings \{i.e., $\Delta / \Gamma \gg$ $\left.0.5\left[\mathrm{I}\left(\mathrm{r}_{\mathrm{t}}\right) / \mathrm{I}_{\mathrm{s}}\right]^{1 / 2}\right\}$ the position-dependent acceleration is given by $\mathrm{a}_{\mathrm{sc}}(\mathrm{r})=(\pi / 4) \nu_{\mathrm{rec}} \Gamma\left[\mathrm{I}(\mathrm{r}) / \mathrm{I}_{\mathrm{s}}\right](\Gamma / \Delta)^{2}$, where $\Gamma$ $(\approx 5 \mathrm{MHz})$ is the natural linewidth, $I(r)$ is the intensity of the guide beam at a distance $r$ from the center of the core $\left(r_{t}\right.$ is the atoms' turning point or maximum range), $I_{s}$ is the saturation intensity $\left(\approx 1.1 \mathrm{~mW} / \mathrm{cm}^{2}\right.$ for Cs), $\Delta$ is the detuning from resonance, and $\nu_{\text {rec }}$ $(\approx 0.4 \mathrm{~cm} / \mathrm{s})$ is the recoil velocity. The center of mass accelerates with a mean acceleration that we obtain by averaging this position-dependent acceleration over the atomic ensemble in time. In the one-dimensional case, in which the atoms are restricted to radial mo- tion, we find that $\left\langle\mathrm{a}_{\mathrm{sc}}\right\rangle_{\text {ensemble }} \propto 1 / \sqrt{\Delta}$ to first order, in qualitative agreement with our observations. For the conditions of Fig. $2\left(\Delta \approx 1.5 \mathrm{GHz}, \mathrm{T}_{\text {MOT }} \approx 80 \mu \mathrm{K}\right)$, our model predicts $\sim 1200$ absorptions per second and an optical acceleration of $\sim 5 \mathrm{~m} / \mathrm{s}^{2}$. This agreement with our observations suggests that our one-dimensional model captures most of the dynamics between the atoms and the tunnel.

We monitored the atoms in the tunnel for times well beyond $35 \mathrm{~ms}$ with images in the longitudinal direction [Fig. 2(b)]. This was possible by combination and separation of the tunnel and the shadow beams with beam splitters. Before we analyze Fig. 2(b), a few comments are in order. First, the bright circle around the atom cloud at $20 \mathrm{~ms}$ is the guide wall. Consequently, the diameter of the cloud changes substantially from 0 to $20 \mathrm{~ms}$ because an appreciable number of

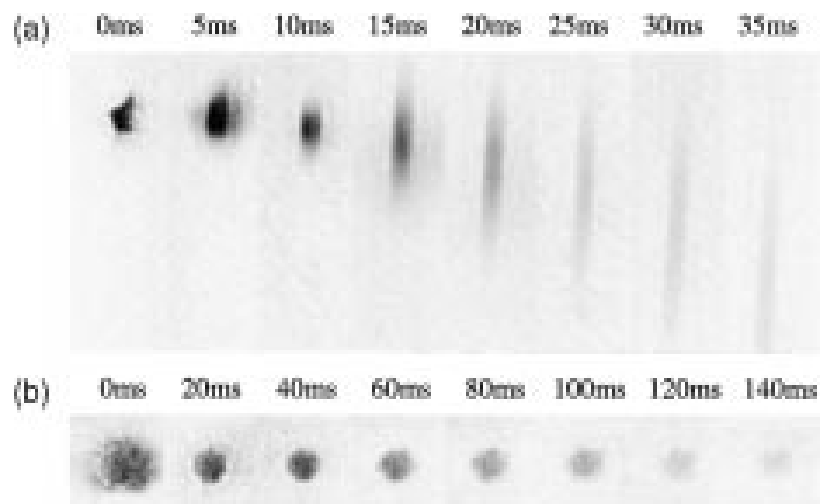

Fig. 2. Shadow images of the atom cloud in the all-light guide: (a) transverse view of the cloud from 0 to $35 \mathrm{~ms}$ without the repumping beam and (b) Iongitudinal view from 0 to $150 \mathrm{~ms}$ with the repumping beam. In (a), the center of mass accelerates at $\sim 15 \mathrm{~m} / \mathrm{s}^{2}$. In both cases the tunnel beam was directed parallel to gravity and detuned $\sim 1.5 \mathrm{GHz}$ to the blue of resonance.

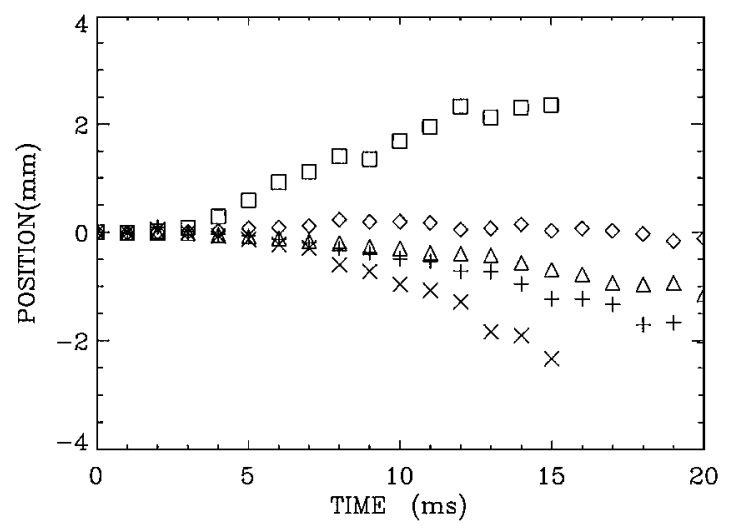

Fig. 3. Position of the center of mass of the cloud versus time at various detunings and orientations of the tunnel beam relative to gravity. The top three traces represent the tunnel beam antiparallel to gravity with detuning: ( $\square) 0.25 \mathrm{GHz},(\diamond) 1 \mathrm{GHz},(\triangle) 1.75 \mathrm{GHz}$. The bottom two traces represent the tunnel beam parallel to gravity with detuning: (+) $1.75 \mathrm{GHz},(\times) 0.25 \mathrm{GHz}$. The magnitude of the uncertainty in these measurements is approximately the size of the symbols. 


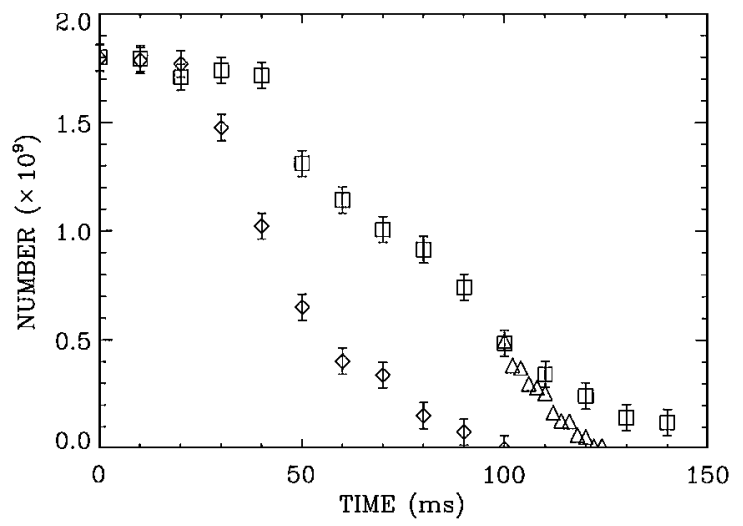

Fig. 4. Number of atoms in the tunnel versus time from longitudinal images [similar to Fig. 2(b)] ( $\square$ ) with the repumper, $(\diamond)$ without the repumper, and $(\triangle)$ in the presence of the repumper but with the kicker beam placed $10 \mathrm{~cm}$ bel ow the MOT.

the atoms in the MOT are outside the tunnel. Second, we optimized the images for long times, so absorption during the first $40 \mathrm{~ms}$ was saturated. Third, we stopped taking images at $150 \mathrm{~ms}$ because the atoms hit the bottom chamber window after $140 \mathrm{~ms}, 18 \mathrm{~cm}$ be low the MOT, and quickly disappeared. To reach the bottom, however, an auxiliary repumping beam that is split from the MOT repumping beam must copropagate with the tunnel beam to keep the atoms in the proper hyperfine level. Figure 4 shows the number of atoms in the tunnel as a function of time. With the repumper, $\sim 10^{8}$ atoms make it to the bottom of the chamber, whereas without the repumper we detect no atoms at the bottom. As an independent check that the atoms remain in the tunnel, we introduced a $50-\mathrm{mW} / \mathrm{cm}^{2}$ kicking beam $\sim 10 \mathrm{~cm}$ bel ow the MOT to knock the atoms out of the tunnel before they hit the window. As the triangles in Fig. 4 show, nearly all the atoms are kicked out of the beam before hitting the window. It is interesting to note that the acceleration deduced for the flight time to the kicker beam is $15-16 \mathrm{~m} / \mathrm{s}^{2}$, in agreement with the transverse measurements at early times.

Finally, we point out that the potential depth, $U(r)=$ $\pi \overline{\mathrm{h}} \Delta \operatorname{In}\left\{1+\left[\mathrm{I}(\mathrm{r}) / \mathrm{I}_{\mathrm{s}}\right]\left[1+4(\Delta / \Gamma)^{2}\right]^{-1}\right\},{ }^{10}$ of the guide in Fig. 1 is $\sim 1 \mathrm{mK}$. This is more than 10 times the temperature of the atoms during their flight. As mentioned above, the initial temperature in this case is $80 \mu \mathrm{K}$, which leads to an absorption rate of $1200 \mathrm{~s}^{-1}-$ each atom absorbs and emits 180 photons on average during the 150-ms flight. Emission, however, heats the ensemble by only $\sim 15 \mu \mathrm{K}$. The primary tunnel loss mechanism is associated with collisions with the warm Cs atoms and not an inadequate guide potential. We placed our guide in a relatively high-pressure environment, $1.3 \times 10^{-7}$ Torr, to enhance the number of atoms trapped in the MOT. At this pressure, the half-life as a result of these collisions is estimated to be $\sim 30 \mathrm{~ms}^{11}$ consistent with what we observe in Fig. 4. Although only $\sim 3 \%$ of the atoms make it through the tunnel in Fig. $4(\Delta \approx 1.5 \mathrm{GHz})$, when we reduced the detuning to $\sim 0.25 \mathrm{GHz}$ to increase the speed of the atoms through the tunnel, effectively reducing the number of collisions, the efficiency was $\sim 10 \%$. To enhance the efficiency further we must reduce the pressure. If the tunnel were placed in a $10^{-8}$-Torr environment, we estimate that the efficiency would increase to $\sim 70 \%$.

In summary, we have demonstrated long-distance transport of ultracold atoms in an all-light guide, using relatively simple optics. We showed that by adjustment of the detuning and the direction of the tunnel beam relative to gravity, it is possible to control the speed and direction of the atoms in the tunnel. Finally, we transported cold atoms for $\sim 20 \mathrm{~cm}$, with an efficiency limited by collisions with warm atoms.

We thank W. C. Degraffenreid, C. R. Orzel, and W. D. Phillips for hel pful discussions and T. Lee and J . Zhu for technical support. This work was supported by the National Science Foundation through grant PHY 9600203. The e-mail address of W. T. Hill III is wth@ipst.umd.edu.

\section{References}

1. J. J . McClelland and M. R. Scheinfein, J. Opt. Soc. Am. B 8, 1974 (1991).

2. G. M. Gallatin and P. L. Gould, J. Opt. Soc. Am. B 8, 502 (1991).

3. S. Kuppens, M. Rauner, M. Schiffer, G. Wokurka, T. Slawinski, M. Zinner, K. Sengstock, and W. Ertmer, in Ultracold Atoms and Bose-Einstein Condensation, K. Burnett, ed., Vol. 7 of OSA Trends in Optics and Photonics Series (Optical Society of America, Washington, D.C., 1996), p. 102.

4. J. Yin, Y. Lin, K. Lee, H. Nha, H. Noh, Y. Wang, K. Oh, U. Paek, and W. J he, J. Korean Phys. Soc. 33, 362 (1998).

5. M. J . Renn, D. Montgomery, O. Vdovin, D. Z. Anderson, C. E. Wieman, and E. A. Cornell, Phys. Rev. Lett. 75, 3252 (1995); M. J . Renn, E. A. Donley, E. A. Cornell, C. E. Wieman, and D. Z. Anderson, Phys. Rev. A 53, R648 (1996).

6. I. Manek, Y. B. Ovchinnikov, and R. Grimm, Opt. Commun. 147, 67 (1998).

7. A. V. Mamaev, M. Saffman, and A. A. Zozulya, Phys. Rev. Lett. 77, 4544 (1996).

8. J. Yin, H. Noh, K. Lee, K. Kim, Y. Wang, and W. J he, Opt. Commun. 138, 287 (1997).

9. C. Wieman, G. Flowers, and S. Gilbert, Am. J. Phys. 63, 317 (1995).

10. A. Ashkin, Phys. Rev. Lett. 40, 729 (1978).

11. This is based on a collision cross section of $2 \times$ $10^{-13} \mathrm{~cm}^{2}$, measured by C. R. Monroe, "Experiments with optically and magnetically trapped cesium atoms," Ph.D. dissertation (University of Colorado, Boulder, Colo., 1992). 